\title{
Muscle-Derived Stem Cells Combined With Nerve Growth Factor Transplantation in the Treatment of Stress Urinary Incontinence in Rats
}

\section{Jianxiong Fang}

Guangdong Provincial People's Hospital https://orcid.org/0000-0001-6005-6880

Haosheng Liu

Guangdong Provincial People's Hospital

Tianqi Liu

Guangdong Provincial People's Hospital

Zhenhui Zhang

Guangdong Provincial People's Hospital

Chao Zhao

Guangdong Provincial People's Hospital

Tianming Peng

Guangdong Provincial People's Hospital

\section{Yong Li}

Guangdong Provincial People's Hospital

Qianqian Wang

Guangdong Provincial People's Hospital

Hanzhong Chen

Guangdong Provincial People's Hospital

\section{Teng Li}

Guangdong Provincial People's Hospital

\section{Shang Huang}

Guangdong Provincial People's Hospital

Xiaoyong Pu ( $\sim$ pxyurol@163.com )

Guangdong Provincial People's Hospital

Jiumin Liu

Guangdong Provincial People's Hospital

\section{Research}

Keywords: muscle-derived stem cells, nerve growth factor, gene transplantation, stress urinary incontinence in rats 
Posted Date: November 6th, 2020

DOI: https://doi.org/10.21203/rs.3.rs-100229/v1

License: (c) (i) This work is licensed under a Creative Commons Attribution 4.0 International License. Read Full License 


\section{Abstract}

Objective: To investigate the efficacy of muscle-derived stem cells (MDSCs) combined with nerve growth factor (NGF) in the treatment of stress urinary incontinence (SUI) in rats.

Methods: MDSCs were isolated and extracted from 90 SD rats, and the stem cell characteristics of the cells were identified using flow cytometry. NGF overexpression plasmid was coated with adenovirus and qRT-PCR was applied to verify adenovirus transfection efficiency. The rat models of SUI were constructed and randomly divided into five groups: control group, PBS group, MDSCs+oe-NGF group, MDSCs+vector group, and MDSCs group. After 8 weeks of feeding, the effect of MDSCs combined with NGF on repairing $\mathrm{SUl}$ in rats was detected by measuring the leakage point pressure (LPP) rats and Masson staining of rat urethral sections. The expression of NGF and VEGF was detected by western blot and IHC staining.

Results: Flow cytometry results showed that the isolated MDSCs had stem cell characteristics and good purity. NGF mRNA was significantly upregulated in MDSCs after transfection with adenovirus coated with NGF plasmid. Compared with the control group, the LPP and the ratio of muscle fibers/collagen fibers were significantly increased in the MDSCs treated groups, with the highest increase in the MDSCs + oeNGF group. Western blot and IHC results showed that compared with the control group, the expression of NGF and VEGF in the urethral tissues in the MDSCs treated groups were significantly up-regulated, with the highest increase in the MDSCs + oe-NGF group.

Conclusion: MDSCs alone can improve SUI in rats, while MDSCs combined with NGF is more effective in the treatment of SUI rats by up-regulating VEGF.

\section{Introduction}

Stress urinary incontinence (SUI) is defined by the International Continence Society (ICS) ${ }^{[1]}$ as the involuntary and uncontrolled urine excretion on a sudden increase in abdominal pressure (such as exertion, physical activity, sneezing, or coughing, etc.). At the 6th International Urinary Control Forum [2, 3], a global epidemiological survey of SUI pointed out that $25-45 \%$ of women have had urinary incontinence, $50 \%$ of which are SUI. Because many women with SUI are reluctant to admit the disease or even seek medical attention negatively, the actual incidence of female SUI may reach more than $80 \%$.

Conservative management or surgery is usually applied in the treatment of SUI ${ }^{[4]}$. The former is often used for the frail elderly patients or patients with mild or moderate SUI but unwilling to receive surgery. Conservative management includes weight loss, pelvic floor muscle training (PFMT), drug therapy represented by estrogen, electrical stimulation therapy, intravaginal devices. This treatment has been shown to be beneficial, but it is required good compliance of patients. Surgery, at present, is still recommended as first-line treatment for severe SUI patients with obvious symptoms or patients fail to conservative management. Surgery can restore the normal physiological anatomy of urethra or simulates its mechanical structure as much as possible, at least the anatomy and functional reconstruction of the middle and upper urethra. In addition, the therapeutic filler can also be injected into the bladder neck, 
middle and upper urethra or periurethral structural tissues (such as anterior vaginal wall) of patients through urethra or vagina, so as to reduce the urethral lumen of SUI patients, thereby increasing the resistance of posterior urethra, prolonging the length of functional urethra and improving urinary continence. The existing research results have revealed that surgery has higher success rate in the treatment of SUI compared with the injection therapy, but the corresponding complication rate of surgery is also higher. The development of urethral filler and injection therapy for SUI is rapid, and this treatment has good short-term results but poor long-term effect, with various side effects ${ }^{[5]}$.

Regenerative medicine ${ }^{[2]}$ usually refers to the use of biological and engineering methods to re-create and repair tissues and organs with functional injury or loss, which makes modern medicine treatment gradually developing towards repairing, reconstructing, replacing damaged organ tissues. Stem cells can differentiate into a range of cell types and have the ability to continuously self-renew and self-

sustenance. They can differentiate into progenitor cells to replace apoptotic senescent cells ${ }^{[6]}$. Due to the poor long-term effect of urethral filler injection, another therapeutic approach have attracted interest of academia, which refer to the reconstruction of urethral sphincter function in SUI patients using the regenerative characteristics of stem cells regenerative, thus promoting many related animal experiments ${ }^{[7-10]}$. In these studies, mesenchymal stem cells (MSCs), a source of cells, are usually derived from muscle, adipose tissue, or bone marrow tissue ${ }^{[11]}$. Regardless of where they derived from, MSCs share some common characteristics, such as stable replication potential and self-renew ability, as well as multipotential differentiation. Studies have shown that MSCs is important in SUI repair. For example, Jacques Corcos ${ }^{[12]}$ showed that intrasphincteric injection of MSCs improves repair the damaged external urethral sphincter and had therapeutic effects on animal models of SUI. Stem cells derived from different tissues have their own advantages and disadvantages. For example, muscle-derived stem cells (MDSCs) have a wide range of sources, are easy to obtain, with mature in vitro amplification techniques. Meanwhile, transplant immune rejection of MDSCs has been solved. Muscle fibers obtained after differentiation of MDSCs automatically integrate into the patient's muscle cell group, avoiding the allergic reaction after MDSCs transplantation; and because the low expression of MHC-1, the incidence of immune rejection is further reduced ${ }^{[13-15]}$.

Nerve growth factor (NGF) ${ }^{[16]}$ is a protein with a molecular weight of about 140,000 , which plays an important role in regulating the growth, differentiation and regeneration of nerve cells. With the deepening of research, NGF has found to have biological effects on non-neural cells ${ }^{[17,18]}$, such as promoting the formation of new blood vessels ${ }^{[19]}$, guiding the cell differentiation of damaged muscle spindles ${ }^{[20]}$, and promoting the repair of fibroblasts in the skin, lung and other parts. In addition, it has been found that NGF is also able to promote the differentiation of stem cells ${ }^{[21]}$, which provides a theoretical basis for NGF combined with stem cell transplantation in the treatment of related diseases. Based on these findings, rat models of SUI was constructed in this study to investigate the efficacy of MDSCs combined with NGF in the treatment of SUI, as well as the repair of leak point pressure (LPP) and urethral smooth muscle in rats, so as to explore the application prospect of MDSCs in the treatment of SUI. 


\section{Materials And Methods}

\subsection{Collection and passage of MDSCs}

The method proposed by Burhan Gharaibeh ${ }^{[22]}$ was adjusted for the primary isolation of rat MDSCs. Sprague Dawley (SD) rats were euthanized by cervical dislocation. Under aseptic conditions, about $5 \mathrm{~g}$ of limb muscle was taken. Subsequently, the muscle tissues were digested with $0.2 \%$ collagenase type XI of equal volume to the tissues for 1 hour, $0.2 \%$ dispase for $30 \mathrm{~min}$, and $0.25 \%$ trypsin-0.01\% EDTA for 30 min. After digestion, they were incubated at $37{ }^{\circ} \mathrm{C}$ with $5 \% \mathrm{CO}_{2}$. The culture medium was DMEM-LG with $10 \% \mathrm{FBS}, 10 \% \mathrm{HS}$ and $1 \%$ penicillin/streptomycin. After 24 hours, the culture medium containing non-adherent cells was transferred to a centrifuge tube for centrifugation (1000 rpm, $5 \mathrm{~min}$ ). The cell precipitation was resuspended with fresh culture solution and inoculated into a new culture flask, which was recorded as the first preplate cells (PP1). By that analogy, the cells were transferred to new flasks with new culture solutions at an interval of 24 hours, which were labeled as PP2 to PP6, respectively. PP6 cells are primary MDSCs. After about 7 days of culture, the cells with $80 \%$ confluency were digested using trypsin, and passaged at a ratio of 1:2, and the culture medium was changed every 2 days.

\subsection{Flow cytometry}

PP6 cells were collected and digested using trypsin. After digestion, the cells were centrifuged to take the cell precipitation. The cell precipitation was mashed and resuspended with PBS to adjust the cell concentration to $10^{7} / \mathrm{ml}$, and then incubated with CD34 and CD45 antibodies. The positive rates of CD34 and CD45 were detected by flow cytometry. The experiment was repeated three times.

\subsection{Adenovirus transfection and grouping}

In our previous experiment, adenovirus packaging as well as the construction, amplification, identification and preservation of adenoviral vector carrying NGF were successfully completed. The third passage of MDSCs was inoculated in 6-well plates at a density of $5 \times 10^{5}$ cells/well; then they were divided into three groups: control group, vector group, and oe-NGF group. In the control group, $2 \mathrm{~mL}$ complete culture medium was added; in the vector group, unloaded adenoviral vector supernatant was added; in the oeNGF group, recombinant adenoviral vector solution with high expression of NGF was added. The transfection was conducted according to the instructions, and there are two parallel wells in each group.

\section{4 qRT-PCR}

Total RNA was extracted using Trizol and the RNA purity was determined. The RNA was reverse transcribed into cDNA using M-MLV (Takara, Japan). qRT-PCR reaction was performed on an Applied Biosystems 7500 real-time PCR system (Foster, USA) using a qPCR kit (Takara, Japan). The reaction conditions were as follows: $95^{\circ} \mathrm{C}, 30 \mathrm{~s} ; 95^{\circ} \mathrm{C}, 5 \mathrm{~s} ; 60^{\circ} \mathrm{C}, 34 \mathrm{~s} ; 40$ cycles. The internal reference gene was $\mathrm{GAPDH}$, and the relative expression of NGF was calculated using the $2^{-\triangle \triangle \mathrm{Ct}}$ method. The primers used were synthesized by Guangzhou Nuoguang Biotechnology Co., Ltd.. The primer sequences are shown in Table 1. 
Table 1

Primer sequences

\begin{tabular}{|lll|}
\hline Primer name & \multicolumn{2}{l|}{ Primer sequence $\mathbf{( 5}^{\mathbf{5}^{\prime}} \mathbf{3}^{\mathbf{}} \mathbf{)}$} \\
\hline NGF & $\mathrm{F}$ & CCAGAGATGGTACGCATGGTG \\
\cline { 2 - 3 } & $\mathrm{R}$ & TTTAGCAGGGATGTGACCTCC \\
\hline GAPDH & $\mathrm{F}$ & TGTGGGCATCAATGGATTTGG \\
\cline { 2 - 3 } & $\mathrm{R}$ & ACACCATGTATTCCGGGTCAAT \\
\hline
\end{tabular}

\subsection{Establishment and grouping of rat models of SUI}

The SUI rat model was established by transecting the pudendal nerve and the nerve innervating the iliococcygeus muscle ${ }^{[23]}$. Ninety SD female rats of the same batch and same age were selected. They were anesthetized with intraperitoneal injection of $1 \%$ pentobarbital sodium $(0.3 \mathrm{~mL} / 100 \mathrm{~g})$ followed by skin preparation in sacrococcygeal region. The limbs of the rats were fixed, and then a 1 to $1.5 \mathrm{~cm}$ incision was made longitudinally in the middle of the sacrococcygeal region. The left subcutaneous tissue of the rats was separated to expose the pudendal nerve, and the pudendal nerve was freed, cut, and removed; subsequently the left sacrococcygeal muscle group was sutured with No.0 silk suture. The right side was treated in the same way. Finally, abdominal skin of the rats was sutured with No.4 silk suture. After modeling, the rats were raised in a routine way for 2 weeks; then their LPP was measured. The LPP of the rats before and after modeling was compared, with decreased LPP as positive. The positive rats were used for subsequent experiments.

The positive rats were divided into five treatment groups (15 rats/group): control group, PBS group, MDSCs + oe-NGF group, MDSCs + vector group, MDSCs group. The peritoneum was opened after anesthesia to expose the bladder, then the bladder was gently pulled and lifted, and the entire urethra was separated downward. Subsequently, the injection process at the juncture of bladder neck and urethral was simulated in the control group, but no solution was injected; in the other groups, the rats were injected with $100 \mu \mathrm{l}$ PBS solution, $100 \mu \mathrm{l}$ NGF gene-modified MDSCs solution, $100 \mu$ l unloaded adenovirus-transfected MDSCs solution, and $100 \mu \mathrm{l}$ prepared MDSCs solution at the juncture of bladder neck and urethral (about $0.5 \mathrm{~cm}$ from the bladder neck) at four points $(3,6,9$, and 12$)$, respectively. After 8 weeks of treatment, urethral tissues were collected from rats in each group; one part of the tissues was fixed for pathological examination, and the other part was rapidly placed in a freezer at $-80^{\circ} \mathrm{C}$.

\subsection{LPP detection}

The rats were anesthetized with intraperitoneal injection of $1 \%$ amobarbital $(0.3 \mathrm{ml} / 100 \mathrm{~g})$. With the simulation of urodynamic test, a ureteral catheter was inserted retrogradely through the external urethral meatus. After the bladder was emptied, a manometric tube was fixed into the bladder, which was connected to the functional experiment pressure sensor through a three-way tube. In addition, the other end of the three-way tube was connected to an extension tube and a syringe and connected to a microinfusion pump. The parameters were set, with $37^{\circ} \mathrm{C}$ normal saline as perfusion solution and a 
perfusion rate of $1 \mathrm{ml} / \mathrm{min}$. When urine appeared in the urethral meatus, the pressure was recorded, which was the LPP. LPP measurement was performed three times before the treatment and at the 8th week after the start of treatment, a total of six times. The average value of the three measurements at each time was recorded.

\subsection{Western Blot}

The fresh urethral tissues of the rats were cut and placed in a mortar. The tissues were homogenized and the total protein was extracted using RIPA lysate. The protein concentration was determined using a BCA protein assay kit (Beyotime, Shanghai, China). Equal concentration of proteins were separated using SDSPAGE and then eletrophoretically transferred to a PVDF membrane. Following the blocking step with 5\% skim milk power for 1 hour at room temperature. Subsequently, the membrane was incubated in a refrigerator overnight at $4{ }^{\circ} \mathrm{C}$ with the following primary antibodies: NGF (ab52918, 1:1000, abcam, Cambridge, UK), VEGF (ab32152, 1:500, abcam, Cambridge, UK) and $\beta$-actin (ab8226, 1:1000, abcam, Cambridge, UK). After rinsed with TBST buffer, the membrane was incubated with secondary antibodies for $30 \mathrm{~min}$. The target proteins were developed chemiluminescence. The gray values of the bands were analyzed using Image $\mathrm{J}$ software.

\subsection{Masson staining}

The urethral tissues were embedded with paraffin and sectioned at a thickness of 3-4 $\mu \mathrm{m}$ using a microtome. Each tissue section was deparaffinized and was performed with Masson staining. The sections were sequentially placed in Masson staining solution for 10 min followed by rinsing with distilled water; color separation using phosphomolybdotungstic acid for $10 \mathrm{~min}$ followed by staining with aniline blue for $10 \mathrm{~min}$; color separation using $1 \%$ acetic acid solution for $60 \mathrm{~s}$. The sections were dehydrated using alcohol ( $95 \%$ as well as absolute alcohol), cleared using xylene. and mounted using neutral resin dropping neutral and cover slip. Finally, the sections were placed under a microscope to observe histopathological changes.

\subsection{Immunohistochemical staining (IHC)}

NGF and VEGF staining was performed on rat urethral sections using an IHC kit (Zhongshan Goldenbridge Biotechnology Co.,LTD., China). The tissue sections were incubated overnight at $4{ }^{\circ} \mathrm{C}$ with NGF antibody (1:1000) and VEGF antibody (1:500); then they were incubated with secondary antibodies at room temperature in the dark and finally color developed. Photographs were taken under a fluorescence microscope.

\subsection{Statistical analysis}

SPSS 24.0 software was used for statistical analysis. All data were expressed as mean \pm standard deviation (SD). Comparisons between two groups were performed using the t-test. $\mathrm{P}<0.05$ indicated a significant difference.

\section{Results}




\subsection{Isolation and identification of MDSCs and adenoviral transfection}

Primary MDSCs cells were isolated, and the morphology of PP1 to PP6 cells was observed under an inverted phase contrast microscope. Most of the PP1 and PP2 were long spindle-shaped fibroblast-like cells and miscellaneous cells with irregular morphology. PP3 and PP4 were mainly long and short shuttleshaped myocytes and occasionally in round-shaped with strong refraction, and myocytes could fuse into myotubes; the number of fibroblasts-like cells was significantly reduced in PP3 and PP4. In PP5, a large number of short shuttle-shaped muscle satellite cells and some round-shaped MDSCs with strong refraction were observed. (Figs. 1A:PP1-PP5) PP6 cells were round-shaped stem cells with strong refraction, and stem cell spheres were observed (Fig. 1A: PP6). PP6 cells were further identified using flow cytometry. As shown in Fig. 1B, the proportion of cells with positive CD34 was $96.93 \%$ and the proportion of cells with positive CD45 was $93.89 \%$. These results indicated that PP6 cells had the characteristics of stem cells and MDSCs had high purity. To upregulate NGF expression in MDSCs cells, the recombinant adenovirus carrying NGF gene (oe-NGF) were transfected in MDSCs. On the 3rd day after transfection, more than $80 \%$ of MDSCs could be observed to carry enhanced green fluorescent protein (EGFP) ( Fig. 1C) under a fluorescence inverted microscope. And the expression of NGF in MDSCs cells after adenoviral transfection were detected using qRT-PCR. As shown in Fig. 1D, NGF expression was significantly upregulated in the MDSCs + oe-NGF groups compared with the control group $(P<0.01)$.

\subsection{MDSCs combined with NGF improve LPP in rats}

After modeling, the female SD rats were randomly divided into five treatment groups, and the LPP in each group was measured after 8 weeks of routing feeding (Figs. 2A and 2B). Compared with the control group, the LPP of rats in the groups with injection of MDSCs was significantly increased $(P<0.0001)$, while the difference between the PBS group and the control group was not statistically significant. The MDSCs + oe-NGF group had the highest LPP after treatment. Compared with the MDSCs + oe-NGF group, the LPP in the MDSCs + vector group and the MDSCs group was reduced by 23.371 and $24.564 \mathrm{cmH} 20$, respectively. These results suggested that injection of MDSCs improved LLP in rats, while the efficacy was better in the MDSCs + oe-NGF group.

\subsection{MDSCs combined with NGF promote urethral smooth muscle repair in SUI rats}

Urethral smooth muscle repair was further detected by Masson staining. As shown in Fig. 3, no significant difference was identified in the ratio of muscle fibers (red)/collagen fibers (blue) in the PBS group compared with the control group $(P>0.05)$. In the MDSCs treated groups, this ratio was upregulated, with the highest ratio in the MDSCs + oe-NGF group $(P<0.0001)$. These results indicated that MDSCs alone could promote the repair of urethral smooth muscle in SUI rats, while the repair efficacy was better in the MDSCs + oe-NGF group. 


\subsection{MDSCs combined with NGF improve SUI by up- regulating VEGF}

Angiogenesis has a positive effect on improving SUl ${ }^{[24]}$, and NGF has reported to promote the secretion of VEGF in retinal Müller cells ${ }^{[25]}$. So we want to explore whether NGF can improve SUI by up-regulating the expression of VEGF. The protein expression of NGF and VEGF in the urethral tissues of rats in each group was detected using Western blot and IHC, respectively. As shown in Fig. 4A-B, compared with the control group, no significant difference was identified in NGF and VEGF protein expression in the PBS group $(P>0.05)$, while the expression was significantly increased in the MDSCs treated groups, with the highest expression in the MDSCs + oe-NGF group $(P<0.0001)$. The results of $\mathrm{IHC}$ was similar to that of Western blot (Fig. 4C-D). Compared with the control group, the difference in IHC scores in the PBS group was not statistically significant $(P>0.05)$, while the IHC scores in the other treatment groups were increased, with the highest score in the MDSCs + oe-NGF group $(P<0.01)$. These results suggested that MDSCs alone could up-regulate the expression of VEGF, and the up-regulation of NGF expression in MDSCs further promoted the expression of VEGF and thus improving SUI.

\section{Discussion}

Urinary incontinence is a common urinary disease that affects more than 200 million people worldwide. SUl is caused by a combination of multiple factors such as blood vessels, nerves, muscles and even endocrine and metabolism. The traditional treatment for SUI only focuses on the reconstruction of urethral structure, but not pay attention to the physiological and biochemical changes of the intrinsic nerves, blood vessels, of urethra and sphincter and periurethral structures, as well as the role of these changes in the development of the disease.

MDSCs are considered as a potential biomaterial for the treatment of SUI, with myogenic, vasculogenic, and neurogenic potential. Animal and human clinical studies have found that MDSCs only differentiate unidirectionally in normal tissues, while in the injured tissues, its multipotency is activated and it can differentiate into a variety of cell types, contributing to the synchronous reconstruction of systemic or local blood vessels, muscle fibers, and peripheral nerves ${ }^{[26-28]}$. A large number of studies ${ }^{[29-32]}$ have demonstrated the injection of stem cell has the potential to restore the function of urethral sphincter in the treatment of SUI. Meanwhile, periurethral injection of MDSCs for the treatment of SUI have also been applied in clinical trials. Lee ${ }^{[33]}$ showed that in the clinical treatment of SUI women, MDSCs had increased LPP and improved urinary continence function compared with the untreated group. Sharifiaghdas ${ }^{[34]}$ found that urethral injection of autologous MDSCs was effective in the treatment of SUI women; and after the continuous follow-up for 3 years, among the 10 patients who participated in the trial, 7 patients achieved clinical cure or symptom improvement, only 3 patients failed to respond to treatment, and all patients had no postoperative adverse reactions. Relevant studies ${ }^{[12,35]}$ also reached similar conclusions in a short- to medium-term follow-up period. In this study, we similarly found that LPP was increased and urethral smooth muscle was repaired after treatment with MDSCs injection in SUI rats. 
In addition, gene editing technology is used to directionally change the expression of stem cell target proteins, so that the modified cells not only have the differentiation characteristics of stem cells, but also can produce specific factors conducive to tissue regeneration, which may be more valuable for SUI treatment. NGF is an important factor involved in the regeneration and functional repair of injured nerves. It also plays an important non-neuronal function in muscle spindle repair, vascular differentiation, and maintenance of immune cells. Meanwhile, NGF can promote the differentiation of stem cells ${ }^{[21]}$. In our study, MDSCs were transfected with adenoviral vector carrying NGF to induce NGF overexpression. In subsequent animal in vivo experiments, we found that the after the treatment of MDSCs combined with NGF, LPP of the SUI rats was significantly elevated, and their urinary continence function was improved. The pathological sections showed that in the MDSCs + oe-NGF group, there were better efficacy in repairing urethral smooth muscle.

VEGF is one of the angiogenic factors that stimulates the growth of new blood vessels ${ }^{[36]}$. Newly generated blood vessels are required to provide essential nutrients to the tissues in studies of muscle tissue engineering ${ }^{[37]}$. It has been shown that with the increase of age, the size and function of bioengineered muscles decrease, but their volume and function were improved by VEGF overexpression in in muscle cells, which has a good application prospect for the treatment of SUI ${ }^{[38]}$. In this study, we found that VEGF expression was significantly upregulated in urethral tissue after in vivo injection of MDSCs in SUI rats, while VEGF expression was higher in the MDSCs + oe-NGF group. These results confirmed that MDSCs combined with NGF repaired urethral tissue injury by upregulating VEGF in SUI rats. However, whether it is through sercretion of cytokines or through signaling pathway or other mechanisms needs further studies. This study is also required further clinical trials to provide more theoretical basis on MDSC in the treatment of SUI in China.

\section{Conclusions}

In summary, recombinant MDSCs combined with NGF overexpression showed good therapeutic effects in the SUI rats, which can be used as a potential therapeutic material for tissue regeneration engineering, bringing hope for better treatment of SUI in female. However, further research is needed to explain the mechanism of the early efficacy, safety and tissue reconstruction before translation to clinical trials.

\section{Declarations}

\section{Ethics approval and consent to participate}

Our experiment has been approved by the Research Ethics Committee of Guangdong Provincial People's Hospital(No.GDREC2018221A).

\section{Consent for publication}

Not applicable 
Availability of data and materials

For data availability, please contact the corresponding author.

\section{Competing interests}

The authors declare that the research was conducted in the absence of any commercial or financial relationships that could be construed as a potential conflict of interest.

\section{Funding}

This work was supported by Guangdong Natural Science Foundation (2019A1515012019), Science and technology program of Guangdong Province (2017ZC0275).

\section{Authors' contributions}

The study was designed by LJ and PX. FJ, LH and LT participated in experimental operation. FJ, LH, LT and ZZ participated in data collection, $\mathrm{CH}, \mathrm{HS}, \mathrm{ZC}, \mathrm{PT}$, LY and WQ participated in statistical analysis. FJ LH and LT wrote the draft, LJ and PX corrected the manuscript. All authors read and approved the final manuscript.

FJ, LH and LT contributed equally to this paper, was the co-first author.

\section{Acknowledgements}

We thank all of the members in the lab. We are grateful to the financial support of the Guangdong Natural Science Foundation.

\section{Conflict of interest}

There are no conflicts of interest to declare.

\section{References}

1. Haylen, B. T.; de Ridder, D.; Freeman, R. M.; et al, An International Urogynecological Association (IUGA)/International Continence Society (ICS) joint report on the terminology for female pelvic floor dysfunction. Int Urogynecol J 2010, 21 (1).

2. Hillary, C. J.; Roman, S.; MacNeil, S.; et al, Regenerative medicine and injection therapies in stress urinary incontinence. Nat Rev Urol 2020, 17 (3), 151-161.

3. Abrams, P.; Andersson, K.-E.; Apostolidis, A.; et al, 6th International Consultation on Incontinence. Recommendations of the International Scientific Committee: EVALUATION AND TREATMENT OF URINARY INCONTINENCE, PELVIC ORGAN PROLAPSE AND FAECAL INCONTINENCE. Neurourol Urodyn 2018, 37 (7), 2271-2272. 
4. Lukacz, E. S.; Santiago-Lastra, Y.; Albo, M. E.; et al, Urinary Incontinence in Women: A Review. JAMA 2017, 318 (16), 1592-1604.

5. Kirchin, V.; Page, T.; Keegan, P. E.; Atiemo, K.; et al, Urethral injection therapy for urinary incontinence in women. Cochrane Database Syst Rev 2012, (2), CD003881.

6. Sharma, S.; Gupta, D. K., Tissue Engineering and Stem Cell Therapy in Pediatric Urology. J Indian Assoc Pediatr Surg 2019, 24 (4), 237-246.

7. Zordani, A.; Pisciotta, A.; Bertoni, L.; et al, Regenerative potential of human dental pulp stem cells in the treatment of stress urinary incontinence: In vitro and in vivo study. Cell Prolif 2019, 52 (6), e12675.

8. Zhou, S.; Zhang, K.; Atala, A.; et al, Stem Cell Therapy for Treatment of Stress Urinary Incontinence: The Current Status and Challenges. Stem Cells Int 2016, 2016, 7060975.

9. Lee, C. N.; Jang, J. B.; Kim, J. Y.; et al, Human cord blood stem cell therapy for treatment of stress urinary incontinence. J Korean Med Sci 2010, 25 (6), 813-816.

10. Wu, R.; Huang, C.; Wu, Q.; et al, Exosomes secreted by urine-derived stem cells improve stress urinary incontinence by promoting repair of pubococcygeus muscle injury in rats. Stem Cell Res Ther 2019, $10(1), 80$.

11. Murphy, M. B.; Moncivais, K.; Caplan, A. I., Mesenchymal stem cells: environmentally responsive therapeutics for regenerative medicine. Exp Mol Med 2013, 45, e54.

12. Corcos, J.; Loutochin, O.; Campeau, L.; et al, Bone marrow mesenchymal stromal cell therapy for external urethral sphincter restoration in a rat model of stress urinary incontinence. Neurourol Urodyn 2011, 30 (3), 447-455.

13. Aragón, I. M.; Imbroda, B. H.; Lara, M. F., Cell Therapy Clinical Trials for Stress Urinary Incontinence: Current Status and Perspectives. Int J Med Sci 2018, 15 (3), 195-204.

14. Sariola, H., The neurotrophic factors in non-neuronal tissues. Cell Mol Life Sci 2001, 58 (8), 10611066.

15. Gill, B. C.; Sun, D. Z.; Damaser, M. S., Stem Cells for Urinary Incontinence: Functional Differentiation or Cytokine Effects? Urology 2018, 117.

16. Rocco, M. L.; Soligo, M.; Manni, L.; et al, Nerve Growth Factor: Early Studies and Recent Clinical Trials. Curr Neuropharmacol 2018, 16 (10), 1455-1465.

17. Kashyap, M.; Kawamorita, N.; Tyagi, V.; et al, Down-regulation of nerve growth factor expression in the bladder by antisense oligonucleotides as new treatment for overactive bladder. J Urol 2013, 190 (2), 757-764.

18. Yoshioka, K.; Tanahashi, M.; Takeda, M.; et al, Induction of Bladder Overactivity by Nerve Growth Factor in Testes in Rats: Possible Neural Crosstalk Between the Testes and Urinary Bladder. Low Urin Tract Symptoms 2016, 8 (1), 62-67.

19. Raza, C.; Riaz, H. A.; Anjum, R.; et al, Repair strategies for injured peripheral nerve: Review. Life Sci $2020,243,117308$. 
20. Sekiya, S.; Homma, S.; Miyata, Y.; et al, Effects of nerve growth factor on differentiation of muscle spindles following nerve lesion in neonatal rats. J Neurosci 1986, 6 (7), 2019-2025.

21. Zhang, L.; Li, X.; Lin, X.; et al, Nerve growth factor promotes the proliferation of Müller cells cocultured with internal limiting membrane by regulating cell cycle via Trk-A/PI3K/Akt pathway. BMC Ophthalmol 2019, 19 (1), 130.

22. Gharaibeh, B.; Lu, A.; Tebbets, J.; et al, Isolation of a slowly adhering cell fraction containing stem cells from murine skeletal muscle by the preplate technique. Nat Protoc 2008, 3 (9), 1501-1509.

23. Wang, H.Y.; Du, X.W.; Xu, J.W.; et al, Establishment and evaluation of an animal model of stress urinary Incontinence. J Clin Rehabil Tissue Eng Res 2010;74:1959-62.

24. Wu, A. K.; Zhang, X.; Wang, J.; et al, Treatment of stress urinary incontinence with low-intensity extracorporeal shock wave therapy in a vaginal balloon dilation induced rat model. Transl Androl Urol 2018, 7 (Suppl 1).

25. Wang, J.; He, C.; Zhou, T.; et al, NGF increases VEGF expression and promotes cell proliferation via ERK1/2 and AKT signaling in Müller cells. Mol Vis 2016, 22, 254-263.

26. Cui, L.; Meng, Q.; Wen, J.; et al, A Functional Comparison of Treatment of Intrinsic Sphincter Deficiency with Muscle-Derived and Adipose Tissue-Derived Stem Cells. IUBMB Life 2018, 70 (10), 976-984.

27. Stangel-Wojcikiewicz, K.; Jarocha, D.; Piwowar, M.; et al, Autologous muscle-derived cells for the treatment of female stress urinary incontinence: a 2-year follow-up of a Polish investigation. Neurourol Urodyn 2014, 33 (3), 324-330.

28. Furuta, A.; Jankowski, R. J.; Pruchnic, R.; et al, The potential of muscle-derived stem cells for stress urinary incontinence. Expert Opin Biol Ther 2007, 7 (10), 1483-1486.

29. Turco, M. P.; de Souza, A. B.; de Campos Sousa, I.; et al, Periurethral muscle-derived mononuclear cell injection improves urethral sphincter restoration in rats. Neurourol Urodyn 2017, 36 (8), 2011-2018.

30. Wang, Z.; Wen, Y.; Li, Y. H.; et al, Smooth Muscle Precursor Cells Derived from Human Pluripotent Stem Cells for Treatment of Stress Urinary Incontinence. Stem Cells Dev 2016, 25 (6), 453-461.

31. Liu, Z.; Cao, Y.; Liu, G.; et al, p75 neurotrophin receptor regulates NGF-induced myofibroblast differentiation and collagen synthesis through MRTF-A. Exp Cell Res 2019, 383 (1), 111504.

32. Kim, S.O.; Na, H. S.; Kwon, D.; et al, Bone-marrow-derived mesenchymal stem cell transplantation enhances closing pressure and leak point pressure in a female urinary incontinence rat model. Urol Int 2011, 86 (1), 110-116.

33. Lee, J.Y.; Cannon, T.W.; Pruchnic, R.; et al. The effects of periurethral muscle-derived stem cell injection on leak point pressure in a rat model of stress urinary incontinence. Int Urogynecol J Pelvic Floor Dysfunct 2003, 14:31-37.

34. Sharifiaghdas, F.; Tajalli, F.; Taheri, M.; et al, Effect of autologous muscle-derived cells in the treatment of urinary incontinence in female patients with intrinsic sphincter deficiency and epispadias: A prospective study. Int J Urol 2016, 23 (7), 581-586. 
35. Stangel-Wojcikiewicz, K.; Piwowar, M.; Jach, R.; et al, Quality of life assessment in female patients 2 and 4 years after muscle-derived cell transplants for stress urinary incontinence treatment. Ginekol Pol 2016, 87 (3), 183-189.

36. Nomi, M.; Atala, A.; Coppi, P.D.; et al, Principals of neovascularization for tissue engineering. Mol Aspects Med 2002, 23: 463-83

37. Folkman, J.; Hochberg, M., Self-regulation of growth in three dimensions. J Exp Med 1973, 138: 74553

38. Delo, D.M.; Eberli, D.; Williams, J.K.; et al, Angiogenic gene modification of skeletal muscle cells to compensate for ageing-induced decline in bioengineered functional muscle tissue. BJU Int 2008, 102: 878-84.

\section{Figures}

A
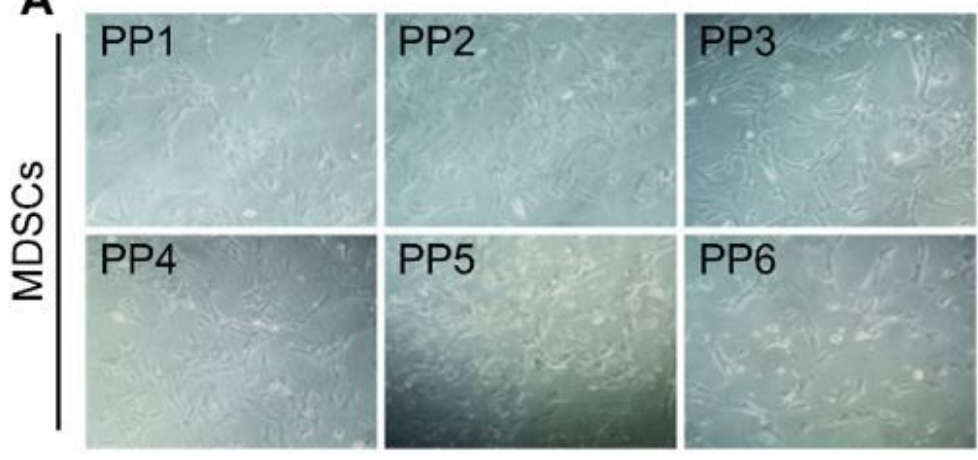

C

MDSCs

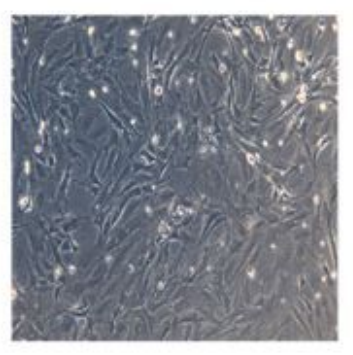

adenovirus-

MDSCs

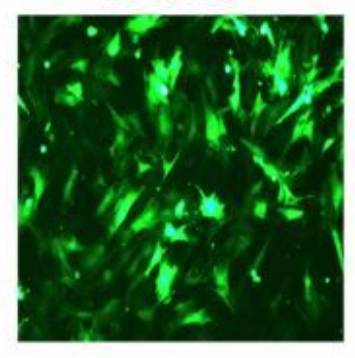

B
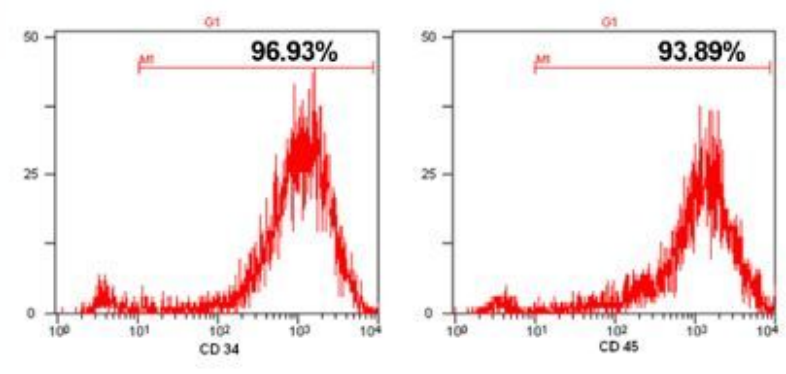

D

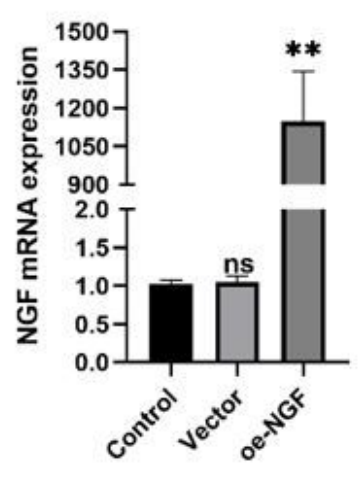

\section{Figure 1}

Isolation and identification of MDSCs and adenoviral transfection. A. After isolation, the morphology MDSCs is observed under an inverted microscope $(200 \mu \mathrm{m})$; B. Flow cytometry to identify MDSCs surface markers CD34 and CD45; C. A fluorescence microscope to observe the transfection efficiency of adenovirus in MDSCs; D. qRT-PCR to detect the expression of NGF in MDSCs transfected with adenovirus. Compared with the control group, ${ }^{* *} \mathrm{P}<0.01$, ns $\mathrm{P}>0.05$. 

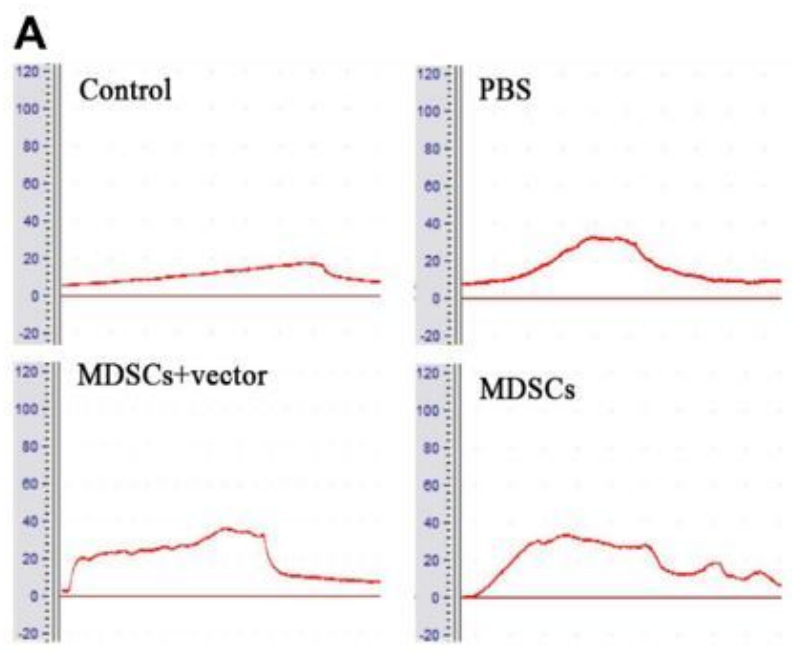

B
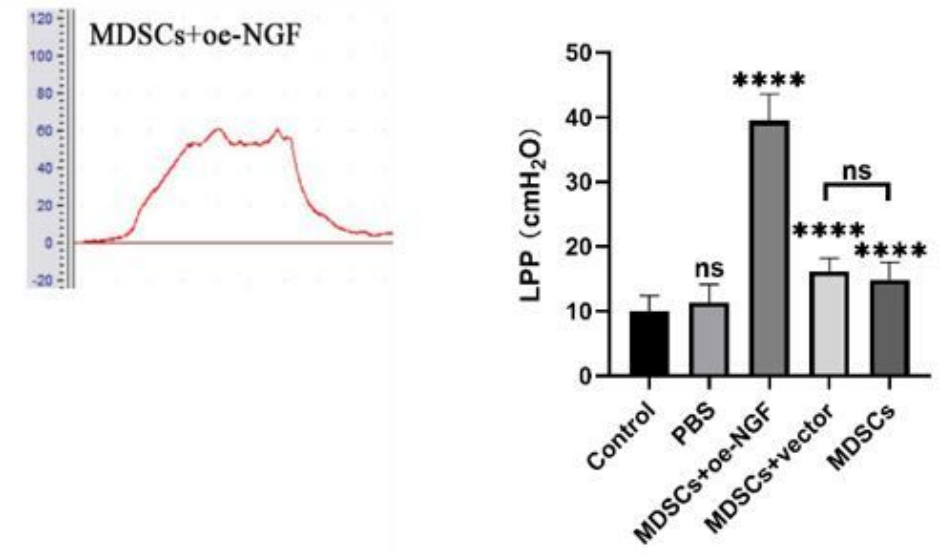

Figure 2

MDSCs combined with NGF improve LPP in rats. A. Oscillograms of LPP in each treatment group; B. Quantification results of LPP in each treatment group. Compared with the control group, ns $P>0.05$, $\star \star \star \star P<0.0001$. 


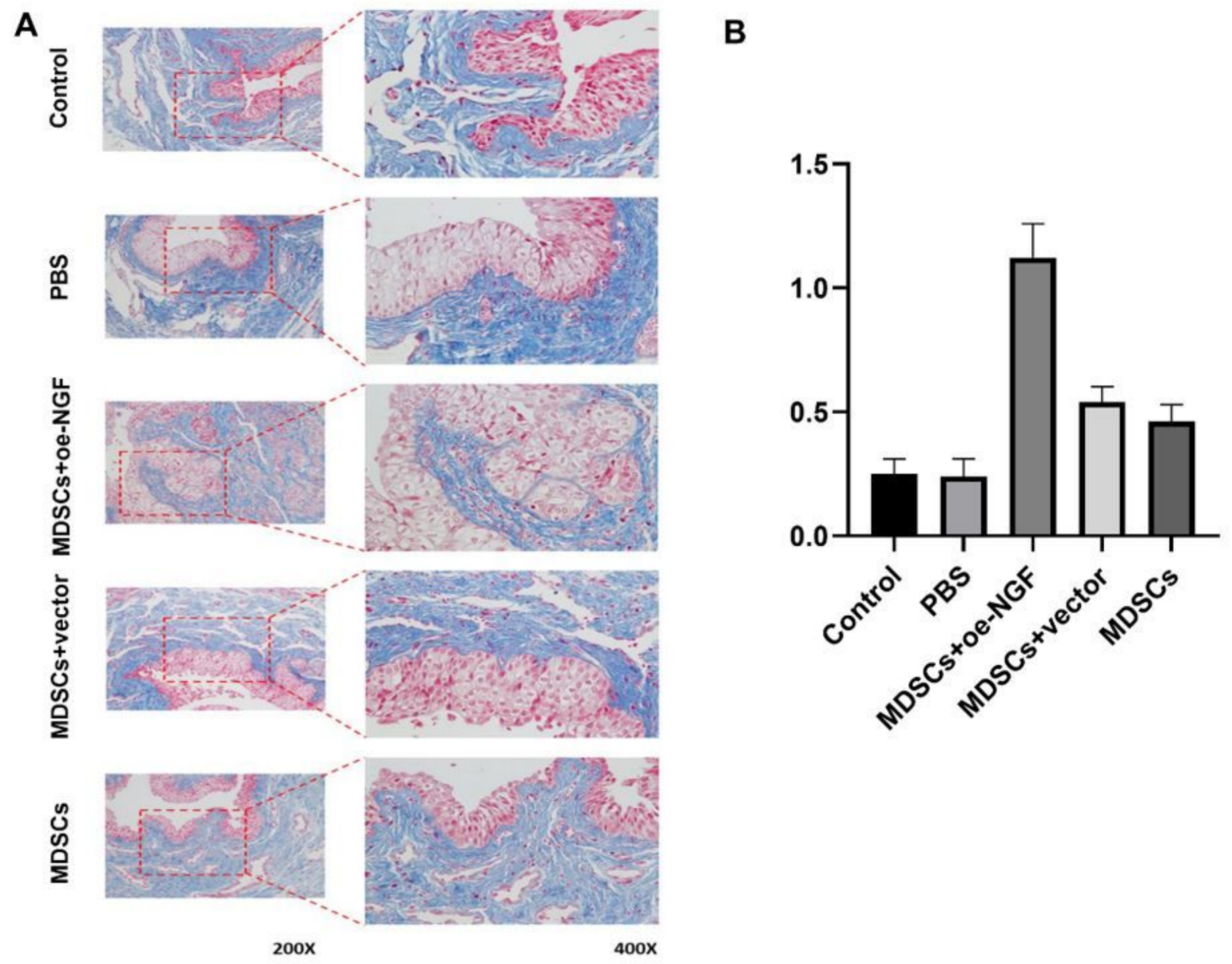

Figure 3

MDSCs combined with NGF promote urethral smooth muscle repair in SUI rats. A. Masson staining of urethral tissues in rats in each treatment group; B. Quantitative analysis results of Masson staining. The area ratio of muscle fibers (red) to collagen fibers (blue) is used for quantitative analysis. Compared with the control group, ns $P>0.05$, ${ }^{\star} P<0.05$ vs. control group, ${ }^{\star *} \mathrm{P}<0.01,{ }^{\star \star \star \star} \mathrm{P}<0.0001$. 
A

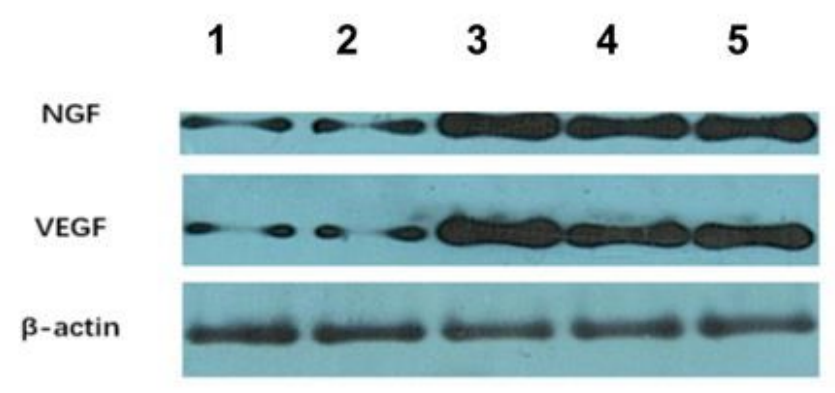

c

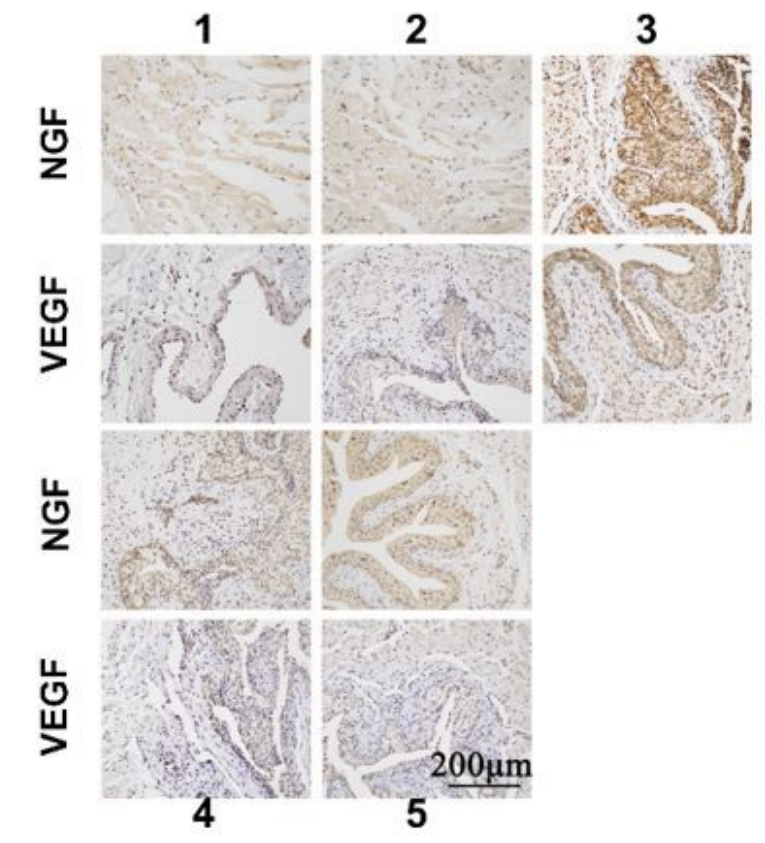

B

- 1. Control ש 3.MDSCs+oe-NGF

口 5. MDSCs

口 2. PBS $\square$ 4. MDSCs+vector

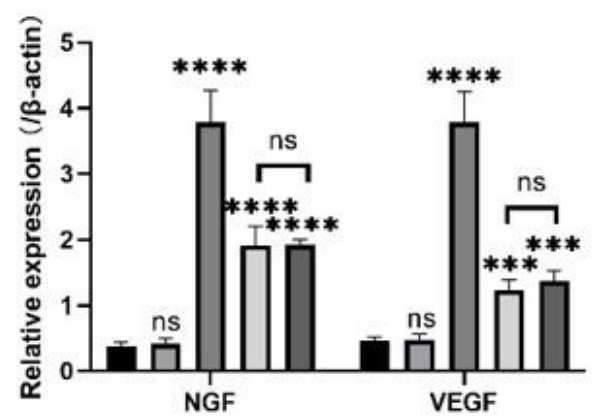

D

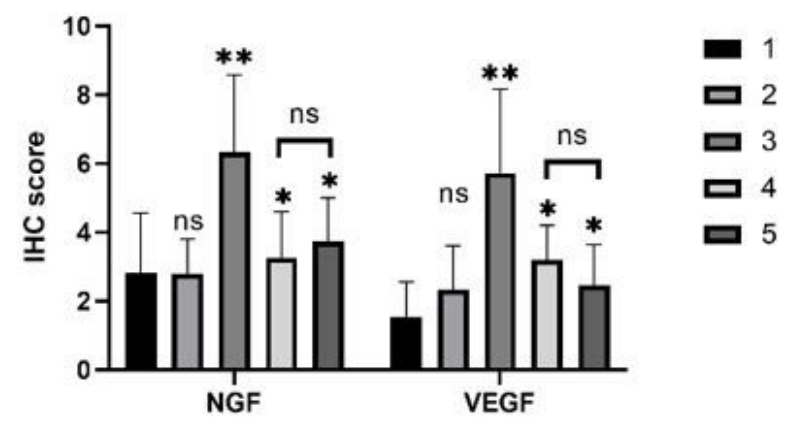

\section{Figure 4}

MDSCs combined with NGF improve SUI by up-regulating VEGF. A. Western blot to detect the expression of NGF and VEGF in the urethral tissues of rats in each treatment group; B. Quantitative analysis of gray values of western blot bands. C. IHC staining results of urethral paraffin sections of rats in each group; D.

Quantitative analysis of $\mathrm{IHC}$ staining results. Compared with the control group, ${ }^{\star * P}<0.01,{ }^{\star *} \mathrm{P}<0.001$, $\star \star \star \star * P<0.0001$, ns $\mathrm{P}>0.05$. 